\title{
Cluster algebras as Hall algebras of quiver representations
}

\author{
Philippe Caldero and Frédéric Chapoton
}

\begin{abstract}
Recent articles have shown the connection between representation theory of quivers and the theory of cluster algebras. In this article, we prove that some cluster algebras of type A-D-E can be recovered from the data of the corresponding quiver representation category. This also provides some explicit formulas for cluster variables.
\end{abstract}

Mathematics Subject Classification (2000). 16G20 16G70 18E30.

Keywords. Cluster algebra, quiver representation, Grassmannian of submodules.

\section{Introduction}

Cluster algebras were introduced in [FZ02] by S. Fomin and A. Zelevinsky in connection with the theory of dual canonical bases and total positivity. Coordinate rings of many varieties from Lie group theory - semisimple Lie groups, homogeneous spaces, generalized Grassmannian, double Bruhat cells, Schubert varieties - have the structure of a cluster algebra, at least conjecturally, see [BFZ05], [Sco06]. One of the goals of the theory is to provide a general framework for the study of canonical bases of these coordinate rings and their $q$-deformations.

A (coefficient-free) cluster algebra $\mathcal{A}$ of rank $n$ is a subalgebra of the field $\mathbb{Q}\left(u_{1}, \ldots, u_{n}\right)$. It is defined by a distinguished set of generators, called cluster variables, constructed by an induction process from an antisymmetrizable matrix $B$, see Section 2.1. The Laurent phenomenon asserts that $\mathcal{A}$ is a subalgebra of $\mathbb{Q}\left[u_{1}^{ \pm 1}, \ldots, u_{n}^{ \pm 1}\right]$. There exists a notion of compatibility between two cluster variables; maximal subsets of pairwise compatible cluster variables are called clusters. All clusters have the same cardinality, which is the rank of the cluster algebra.

A cluster algebra is of finite type if the number of cluster variables is finite. The classification of cluster algebras of finite type [FZ03a] is a fundamental step in the theory. The main result is that these cluster algebras come from an antisymmetrized Cartan matrix of finite type, see Section 2.2. Moreover, in this case the cluster variables are in correspondence with the set of almost positive roots $\Phi_{\geq-1}$, i.e. positive roots or opposed simple roots, of the root system. 
The Gabriel theorem asserts that the set of indecomposable representations of a quiver $Q$ of Dynkin type is in bijection with the set $\Phi_{+}$of positive roots. The cluster category $\mathcal{C}$ was constructed in $\left[\mathrm{BMR}^{+}\right],[\mathrm{CCS} 04]$ as an extension of the category $\bmod _{k}(Q)$ of finite dimensional $k Q$-modules, such that the set of indecomposable objects of $\mathcal{C}$ is in bijection with $\Phi_{\geq-1}$. The category $\mathcal{C}$ is not abelian in general, but it is a triangulated category, [Kel]. In $\left[\mathrm{BMR}^{+}\right]$, this category is studied in depth. The authors give a correspondence between cluster variables and indecomposable objects of $\mathcal{C}$. They prove that the compatibility of two cluster variables corresponds to the vanishing of the Ext groups; hence, clusters correspond to so-called ext-configurations. They prove that there exist many analogies between finite cluster algebras and cluster categories, but the properties of the correspondence are mostly conjectural, see $\left[\mathrm{BMR}^{+}\right.$, Conjecture 9.3].

In [CCS04], the authors prove that the denominators of cluster variables can be calculated from $\mathcal{C}$ in type $\mathrm{A}$. They give a combinatorial/geometric approach of $\mathcal{C}$ in the spirit of Teichmüller spaces, [FG03].

The implicit question behind all articles [MRZ03], [BMR ${ }^{+}$], [CCS04] dealing with cluster algebras and quiver theory is the following: Can one realize the cluster algebra as a "Hall algebra" of the category $\mathcal{C}$ in some sense?

$\operatorname{Recall}$ that $\bmod _{k}(Q)$ is a (non full) subcategory of $\mathcal{C}$. In this article, the cluster variable associated to an indecomposable $k Q$-module is explicitly given, see Theorem 3.4. This result is interesting from different angles.

1. It strengthens the relations between the category $\mathcal{C}$ and the algebra $\mathcal{A}$.

2. We obtain here explicit expressions for cluster variables, instead of inductive ones. These expressions are in terms of Euler-Poincaré characteristic of Grassmannians of submodules. Note that these characteristics can be easily calculated in the $\mathrm{A}_{n}$ case, see Example 3.2. They can also be calculated in a combinatorial way in the $\mathrm{D}_{n}$ case.

3. One important open question in cluster theory is the positivity conjecture [FZ02, $\S 3]$, which says that cluster variables should be Laurent polynomials with positive coefficients in the variables of any fixed cluster. Our explicit expressions will be used in another article $[\mathrm{CK}]$ to show that cluster variables indeed have a positive Laurent expansion in any cluster associated to a Dynkin type quiver. Recall that the positivity conjecture is known to hold only for a distinguished cluster so far [FZ03a, Thm. 1.10].

4. The expression gives the possibility to quantize cluster algebras in the RingelHall algebras spirit: the Euler-Poincaré characteristic should be replaced by a polynomial which counts $\mathbb{F}_{q}$-rational points on the variety.

These points as well as other ones (toric degenerations, denominator theorems) will be developed in a forthcoming article, $[\mathrm{CK}]$, which mainly relies on the cluster variable formula, Theorem 3.4.

In the sequel, we give a conjectural expression for cluster variables associated to a multiplicity-free indecomposable module over any quiver of simply-laced finite 
cluster type. As a special case, this conjecture enables, in $\mathrm{A}_{n}$ type, to calculate in a combinatorial way the cluster variables in terms of any cluster.

To conclude, we give a connection between our theorem, the geometric realization of [CCS04], and the Coxeter-Conway friezes [CC73].

We would like to note that one of the starting points for the experimental work leading to this article was the combinatorial expressions for some $Y$-system Laurent polynomials given in [FZ03b] for multiplicity-free roots. Although these are definitely not the same as cluster Laurent polynomials, the combinatorics is quite similar.

Acknowledgments. The first author would like to thank Markus Reineke for conversations on Euler-Poincaré characteristic and Grassmannians of submodules. He is also grateful to Bernhard Keller and Andrew Hubery for corrections on the proof of Lemma 3.11.

\section{Recollection from cluster algebras and cluster categories}

2.1. In this section, we give basic definitions and theorems concerning cluster algebras, see [FZ02], [FZ03a], [BFZ05]. The cluster algebras in this article are defined on a trivial semigroup of coefficients, and will be called reduced cluster algebras. Let us first recall basic results on cluster algebras, in the special case of reduced cluster algebras.

Let $n$ be a positive integer and let $B=\left(b_{i j}\right)$ be a square matrix in $M_{n}(\mathbb{Z})$. We say that $B$ is antisymmetrizable if there exists a diagonal matrix $D$ in $M_{n}(\mathbb{N})$ such that $D B$ is antisymmetric. We introduce the field $\mathcal{F}:=\mathbb{Q}\left(u_{1}, \ldots, u_{n}\right)$, with algebraically independent generating set $\underline{u}:=\left(u_{1}, \ldots, u_{n}\right)$. A pair $(\underline{x}, B)$, where $\underline{x}=\left(x_{1}, \ldots, x_{n}\right)$ is an algebraically independent generating set of $\mathcal{F}$ and where $B$ is an antisymmetrizable matrix, will be called a seed. In the sequel, we will identify the rows and the columns of the matrix $B$ with the elements of $x$.

Fix a seed $(\underline{x}, B), B=\left(b_{y z}\right)$, and $w$ in the base $\underline{x}$. Let $w^{\prime}$ in $\mathcal{F}$ be such that

$$
w w^{\prime}=\prod_{b_{y w}>0} y^{b_{y w}}+\prod_{b_{y w}<0} y^{-b_{y w}} .
$$

This is the so-called exchange relation. Now, set $\underline{x}^{\prime}:=\underline{x}-\{w\} \cup\left\{w^{\prime}\right\}$ and $B^{\prime}=\left(b_{y z}^{\prime}\right)$ such that

$$
b_{y z}^{\prime}= \begin{cases}-b_{w z} & \text { if } y=w^{\prime} \\ -b_{y w} & \text { if } z=w^{\prime} \\ b_{y z}+1 / 2\left(\left|b_{y w}\right| b_{w z}+b_{y w}\left|b_{w z}\right|\right) & \text { otherwise }\end{cases}
$$


Then, it is known that $\left(\underline{x}^{\prime}, B^{\prime}\right)$ is also a seed. We say that this seed is the mutation of the seed $(\underline{x}, B)$ in the direction $w$. We also say that $w$ and $w^{\prime}$ form an exchange pair. It is easily seen that the mutation of the seed $\left(x^{\prime}, B^{\prime}\right)$ in the direction $w^{\prime}$ is $(\underline{x}, B)$. We can define the equivalence relation generated by $(\underline{x}, B) \sim\left(\underline{x}^{\prime}, B^{\prime}\right)$ if $\left(\underline{x}^{\prime}, B^{\prime}\right)$ is a mutation of $(\underline{x}, B)$.

We assign to an antisymmetrizable matrix $B$ a $\mathbb{Q}$-algebra in the following way.

Definition 2.1. The reduced cluster algebra $\mathcal{A}(B)$ associated to the antisymmetrizable matrix $B$ is the subalgebra of $\mathcal{F}$ generated by all $\underline{x}$ such that $(\underline{u}, B) \sim\left(\underline{x}, B^{\prime}\right)$. Such $\underline{x}$ are called clusters and the elements of $\underline{x}$ are called cluster variables.

Remark 2.2. More generally, see [BFZ05], cluster algebras are associated to rectangular matrices in $M_{n, m}(\mathbb{Z})$. We will not be concerned with such algebras in this article.

Note the so-called Laurent phenomenon, see [FZ02]:

Theorem 2.3. Let $B$ be an antisymmetrizable matrix in $M_{n}(\mathbb{Z})$, then $\mathcal{A}(B)$ is a subalgebra of $\mathbb{Q}\left[u_{i}^{ \pm 1}, 1 \leq i \leq n\right]$.

2.2. This section is concerned with finite reduced cluster algebras, i.e. cluster algebras with a finite number of cluster variables.

Let $\Delta$ be a Dynkin diagram of rank $n$ and let $\mathrm{A}_{\Delta}$ be its Cartan matrix. We denote by $\Phi$, resp. $\Phi_{+}$, the root system, resp. the set of positive roots, associated to $\Delta$. Let $\alpha_{i}$, $1 \leq i \leq n$, be the simple roots and let $\mathcal{Q}$ be the $\mathbb{Z}$-lattice generated by them. We also denote by $\Phi_{\geq-1}$ the set of almost positive roots $\Phi_{+} \cup\left\{-\alpha_{1}, \ldots,-\alpha_{n}\right\}$.

We have the following fundamental theorem, see [FZ03a]:

Theorem 2.4. A reduced cluster algebra $\mathcal{A}$ is finite if and only if there exists a seed $(\underline{x}, B)$ of $\mathcal{A}$ such that the Cartan counterpart of the matrix $B$ is a Cartan matrix of finite type.

In the theorem, the Cartan counterpart of a matrix $B=\left(b_{i j}\right)$ in $M_{n}(\mathbb{Z})$ is the matrix $A=\left(a_{i j}\right)$ with

$$
a_{i j}= \begin{cases}2 & \text { if } i=j, \\ -\left|b_{i j}\right| & \text { if } i \neq j .\end{cases}
$$

Actually, the theorem of Fomin and Zelevinsky is more precise. The correspondence $\mathcal{A} \mapsto \Delta$ of the theorem provides a bijection from the set of finite reduced cluster algebras into the set of Dynkin diagrams of finite type. Hence, to a Dynkin diagram $\Delta$ of type $\mathrm{A}$ to $\mathrm{G}$, we can associate a unique algebra $\mathcal{A}(\Delta)$; this is the reduced cluster algebra of the corresponding type. 
2.3. We present in this section a recollection on quiver representations. We fix a field $k$ which can be either the finite field $\mathbb{F}_{q}$ or the field $\mathbb{C}$ of complex numbers. From now on, let $\Delta$ be a Dynkin diagram of simply laced finite type and $Q$ be a quiver with underlying graph $\Delta$. We index by $I=\{1, \ldots, n\}$ the set of its vertices. We consider the category $\bmod _{k}(Q)$ of finite dimensional $k$-representations of the quiver $Q$ : the objects of $\bmod _{k}(Q)$ are tuples of finite dimensional vector spaces $\left(M_{i}\right)_{i \in I}$ together with tuples of linear maps $\left(M_{\alpha}: M_{i} \rightarrow M_{j}\right)_{\alpha}: i \rightarrow j$, a morphism between the objects $\left(M_{*}\right)$ and $\left(N_{*}\right)$ is a $I$-family of linear maps $M_{i} \rightarrow N_{i}$ such that for any $\alpha: i \rightarrow j$ the diagram

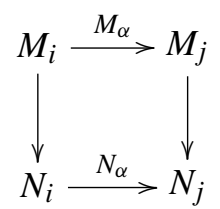

commutes. We denote by $\overline{\bmod }_{k}(Q)$, resp. $\operatorname{Ind}_{k}(Q)$, the set of isoclasses, resp. indecomposable modules, of $\bmod _{k}(Q)$. We also denote by $P_{i}$, resp. $S_{i}$, resp. $I_{i}, 1 \leq i \leq n$, the projective, resp. simple, resp. injective modules of the category. Recall that, by the theorem of Gabriel, the set $\operatorname{Ind}_{k}(Q)$ does not depend on the field $k$ and is in natural bijection with the set of positive roots $\Phi_{+}$. This bijection sends the simple modules $S_{i}$ to the simple roots $\alpha_{i}$. Hence, the Grothendieck group of the category $\bmod _{k}(Q)$ is naturally identified with the lattice $Q$. We define the $\operatorname{map} \underline{\operatorname{dim}}: \bmod _{k}(Q) \rightarrow \mathcal{Q}$ which associates to a module $M$ its class $[M]$ in the Grothendieck group.

We define for $M, N$ in $\bmod _{k}(Q)$ :

$$
\begin{gathered}
{[M, N]=\operatorname{dim} \operatorname{Hom}_{k Q}(M, N), \quad[M, N]^{1}=\operatorname{dim} \operatorname{Ext}_{k Q}^{1}(M, N),} \\
\langle M, N\rangle=[M, N]-[M, N]^{1} .
\end{gathered}
$$

It is known that $\langle$,$\rangle can be defined on the Grothendieck group \mathcal{Q}$; this is the Euler form.

Fix $\underline{e}$ in $\mathcal{Q}$ and let $M$ be in $\bmod _{k}(Q)$. We define the $\underline{e}$-Grassmannian $\operatorname{Gr}_{\underline{e}}(M)_{k}$ of the module $M$ on $\mathrm{k}$ :

$$
\operatorname{Gr}_{\underline{e}}(M)_{k}:=\left\{N \mid N \in \bmod _{k}(Q), N \subset M, \underline{\operatorname{dim}}(N)=\underline{e}\right\} .
$$

When the ground field is clear, we will omit the index $k$ in the notation. Note that $\mathrm{Gr}_{e}(M)$ can be realized as a closed subvariety of the classical Grassmannian $\mathrm{Gr}_{e}(M)$, where $e=\sum_{i} e_{i}$. Hence, the variety $\mathrm{Gr}_{\underline{e}}(M)$ is projective. We define in an obvious way the variety $\operatorname{Gr}_{\underline{e}}(M)$, for $M$ in $\overline{\bmod }_{k}(Q)$, and we set $\operatorname{Gr}(M):=\bigcup_{\underline{e}} \operatorname{Gr}_{\underline{e}}(M)$. So, in the sequel the Grassmannian will always mean "Grassmannian of submodules". 
2.4. The cluster category $\mathcal{C}=\mathcal{C}_{\Delta}$ has been introduced in [BMR ${ }^{+}$] and [CCS04]. As proved in these articles, the cluster category $\mathcal{C}_{\Delta}$ is strongly linked with the finite cluster algebra $\mathcal{A}(\Delta)$. As a first example of this relation, the set of indecomposable objects of $\mathcal{C}_{\Delta}$ is in bijection with $\Phi_{\geq-1}$, so it is in bijection with the cluster variables of $\mathcal{A}(\Delta)$.

Let $Q$ be as above. In the sequel, we denote by $B_{Q}$ be the antisymmetric matrix in $M_{n}(\mathbb{Z})$ such that $b_{i j}=1$ if $i \rightarrow j$ in $Q$ and 0 if $i$ and $j$ are not connected. Let $\mathscr{D} \bmod _{k}(Q)$ be the derived category of $\bmod _{k}(Q)$. Note that as $\bmod _{k}(Q)$ is hereditary, the indecomposable objects of $\mathscr{D} \bmod _{k}(Q)$ are the shifts of $\operatorname{Ind}_{k}(Q)$. We define the functor $F$ of $\mathscr{D} \bmod _{k}(Q)$ by $F: M \mapsto \tau^{-1} S M$, where $S$ is the shift and $\tau$ is the Auslander-Reiten translation. The category $\mathcal{C}_{Q}$ is the category of orbits of $\mathscr{D} \bmod _{k}(Q)$ by $F$, see $\left[\mathrm{BMR}^{+}\right]$. It is a triangulated category [Kel], it is also a Krull-Schmidt category, but it is not abelian in general. A nice property of this category is that the bifunctor Ext ${ }^{1}$ is symmetric. Up to equivalence, the triangulated category $\mathscr{D} \bmod _{k}(Q)$ is well known not to depend on the orientation of $Q$, but only on $\Delta$, by the classical results of Gelfand and Ponomarev. Hence, the category $\mathcal{C}_{Q}$ itself does not depend on the orientation of $Q$, but only on $\Delta$, up to equivalence of triangulated categories. It will be denoted by $\mathcal{C}_{\Delta}$ or just $\mathcal{C}$. The set $\operatorname{Ind}_{k}(Q)$ embeds naturally in the set Ind $\mathcal{C}$ of indecomposable objects of $\mathcal{C}$. Moreover, Ind $\mathcal{C}=$ $\operatorname{Ind}_{k}(Q) \cup\left\{S P_{i}, 1 \leq i \leq n\right\}$. Now, via the theorem of Gabriel, the denominator theorem, [FZ03a], provides a bijection between $\mathrm{Cl}_{\Delta}$ and Ind $\mathcal{C}$.

Let $Q_{\text {alt }}$ be an alternating quiver with underlying diagram $\Delta$, i.e. such that each vertex is either a sink (all arrows incoming) or a source (all arrows outgoing).

Theorem 2.5. Consider the seed $\left(\underline{u}=\left\{u_{1}, \ldots, u_{n}\right\}, B_{Q_{a l t}}\right)$ and a cluster variable $u$, $u \neq u_{i}, 1 \leq i \leq n$. Then, there exists a positive root $\alpha=\sum_{i} n_{i} \alpha_{i}$ such that the denominator of $u$ as an irreducible fraction in $\underline{u}$ is $\prod_{i} u_{i}^{n_{i}}$. The assignment $u \mapsto \alpha$ $\left(u \neq u_{i}\right), u_{i} \mapsto-\alpha_{i}$, provides a bijection from $\mathrm{Cl}_{\Delta}$ to $\Phi_{\geq-1}$.

An important theorem of $\left[\mathrm{BMR}^{+}\right]$asserts that the ext-configurations of Ind $\mathcal{C}$, i.e. maximal subsets of Ind $\mathcal{C}$ with trivial pairwise extension, correspond to clusters via the bijections $\operatorname{Ind}\left(\mathcal{C}_{\Delta}\right) \simeq \Phi_{\geq-1} \simeq \mathrm{Cl}_{\Delta}$. Note also that the ext-configurations of $\operatorname{Ind}_{k}(Q)$ are ext configurations of Ind $\mathcal{C}$ which belong to $\operatorname{Ind}_{k}(Q)$.

2.5. In the previous section, we have seen a correspondence between indecomposable objects of $\mathcal{C}$ and cluster variables. We will see in this section properties of the Auslander-Reiten translation $\tau$ in this correspondence. First of all, let us recall some basic facts on the Auslander-Reiten theory, see [ARS95].

Let $\Gamma_{Q}$ be the Auslander-Reiten quiver of $\bmod _{k}(Q)$. Recall that its set of vertices is $\operatorname{Ind}_{k}(Q)$ and the arrows are given by irreducible morphisms of the category. The AR-quiver $\Gamma_{\mathcal{C}}$ of $\mathcal{C}$ is defined in the same way.

Let $M$ be a non projective module in $\operatorname{Ind}_{k}(Q)$ and let $M^{\prime}=\tau M \in \operatorname{Ind}_{k}(Q)$ be its 
AR-translated. We consider the direct sum $B$ of indecomposable modules $B_{j}$ such that $M^{\prime} \rightarrow B_{j}$ in $\Gamma_{Q}$. Then, $B$ is also the direct sum of indecomposable modules $B_{j}$ such that $B_{j} \rightarrow M$ in $\Gamma_{Q}$ and we have the following exact sequence of modules:

$$
0 \longrightarrow \tau M \longrightarrow B \stackrel{\sigma}{\longrightarrow} M \longrightarrow 0 \text {. }
$$

Moreover, this exact sequence is almost split in the following sense: each morphism $N \rightarrow M$ which is not a split epimorphism factors through $\sigma$.

The AR-quivers of $\bmod _{k}(Q)$ are well known and can be explicitly described, see [Gab80].

The AR-quiver $\Gamma_{\mathcal{C}}$ is a slight extension of $\Gamma_{Q}$. Indeed, see $\left[\mathrm{BMR}^{+}\right]$, each exact sequence as in (7) gives rise in the triangulated category $\mathcal{C}$ to a triangle

$$
\tau M \rightarrow B \rightarrow M \rightarrow S \tau M,
$$

where the first two morphisms are composed with irreducible morphisms. In other words, the embedding $\operatorname{Ind}_{k}(Q) \subset$ Ind $\mathcal{C}$ provides an embedding $\Gamma_{Q} \subset \Gamma_{\mathcal{C}}$ as a full subquiver. In order to describe $\Gamma_{\mathcal{C}}$, it is sufficient to note that:

There exists an arrow $S P_{i} \rightarrow M$ in $\Gamma_{\mathcal{C}}$ if and only if

$$
M= \begin{cases}S P_{k} & k \rightarrow i \text { in } Q, \\ P_{j} & i \rightarrow j \text { in } Q,\end{cases}
$$

and there exists an arrow $M \rightarrow S P_{i}$ in $\Gamma_{\mathcal{C}}$ if and only if

$$
M= \begin{cases}P_{k} & k \rightarrow i \text { in } Q, \\ S P_{j} & i \rightarrow j \text { in } Q .\end{cases}
$$

In the following proposition, we denote by $x_{M}$ the cluster variable corresponding to the indecomposable object $M$ of Ind $\mathcal{C}$.

Proposition 2.6. Let $\mathcal{C}$ be the cluster category of type $A, D$ or $E$.

(i) Let $M$ be an indecomposable object of $\mathcal{C}$. Then $x_{M}$ and $x_{\tau M}$ form an exchange pair.

(ii) With the notation above, we have

$$
x_{\tau M} x_{M}=\prod_{j} x_{B_{j}}+1 .
$$

Proof. (i) is a direct application of [BMR ${ }^{+}$, Proposition 7.6].

Fix $M$ in Ind $\mathcal{C}$. Then, there exists a quiver $Q$ and a sink $i$ of $Q$ such that in

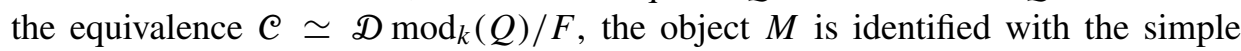


projective $P_{i}=S_{i}$. The algebra $\mathcal{A}(\Delta)$ is by construction isomorphic to $\mathcal{A}\left(B_{Q}\right)$. By the discussion above, see (9) and (10), it is enough to prove that

$$
x_{S P_{i}} x_{S_{i}}=\prod_{i \rightarrow j} x_{S P_{j}}+1 .
$$

Let us prove the equality. Set $B_{Q}=\left(b_{i j}\right)$. The exchange relation gives

$$
x_{S P_{i}} x_{S_{i}}=\prod_{b_{j i}=1} x_{S P_{j}}+\prod_{b_{j i}=-1} x_{S P_{j}} .
$$

As $i$ is a sink of $Q$, the second term is one. Moreover, $b_{j i}=1$ if and only if $j \rightarrow i$ in $Q$. So, we have the claimed equality.

Remark that (ii) is a particular case of $\left[\mathrm{BMR}^{+}\right.$, Conjecture 9.3].

\section{The main theorem}

3.1. In this section, for each quiver $Q$ with underlying Dynkin diagram $\Delta$ of type A-D-E, we realize $\mathcal{A}(\Delta)$ from the module category $\mathcal{C}_{Q}$. Actually, we recover the algebra $\mathcal{A}\left(B_{Q}\right)$ from the category $\mathcal{C}_{Q}$. In the sequel, we fix a quiver $Q$ of type A-D-E.

Recall that $\mathcal{F}=\mathbb{Q}\left(u_{i}, 1 \leq i \leq n\right)$. For each $M$ in $\overline{\bmod }_{k}(Q)$ with dimension vector $\underline{\operatorname{dim}}(M)=\underline{m}=\sum_{i} m_{i} \alpha_{i}$, set

$$
X_{M}=\sum_{\underline{e}} \chi\left(\operatorname{Gr}_{\underline{e}}(M)\right) \prod_{i} u_{i}^{-\left\langle\underline{e}, \alpha_{i}\right\rangle-\left\langle\alpha_{i}, \underline{m}-\underline{e}\right\rangle},
$$

where $\chi$ is the Euler-Poincaré characteristic of the complex Grassmannian. Remark that the sum is finite since the dimension vectors $\underline{e}=\sum_{i} e_{i} \alpha_{i}$ which occur in the sum verify $0 \leq e_{i} \leq m_{i}$. We now illustrate with examples.

Example 3.1. Suppose that $Q$ is the following alternated orientation for $\mathrm{A}_{3}$ :

$$
1 \longrightarrow 2 \longleftarrow 3 \text {. }
$$

Then, the indecomposable modules of $\bmod _{k}(Q)$ are $S_{1}, S_{2}, S_{3}, P_{1}, P_{3}, I_{2}$, where $\left[I_{2}\right]=\left[S_{1}\right]+\left[S_{2}\right]+\left[S_{3}\right]$. The AR-quiver $\Gamma_{\mathcal{C}}$ has the following shape:

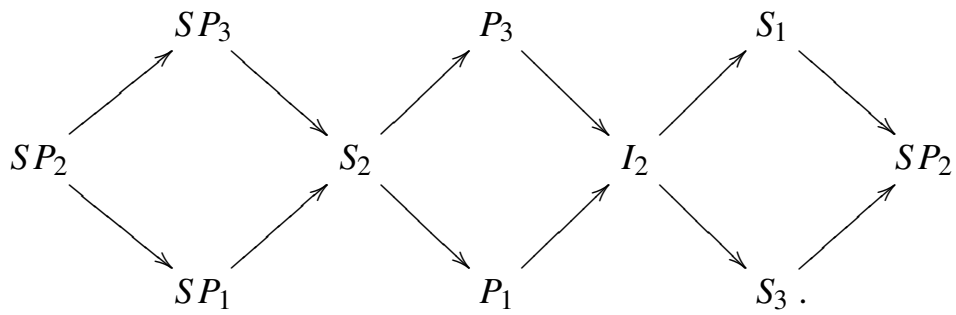


We compute explicitly the $X_{M}$ using formula (14). In the following sums, the terms are ordered by $\sum e_{i}$. The submodules of $S_{2}$ are 0 and $S_{2}$.

$$
X_{S_{2}}=\frac{u_{1} u_{3}}{u_{2}}+\frac{1}{u_{2}}=\frac{u_{1} u_{3}+1}{u_{2}},
$$

The submodules of $P_{3}$ are $0, S_{2}$ and $P_{3}$ and the submodules of $P_{1}$ are $0, S_{2}$ and $P_{1}$.

$$
\begin{aligned}
& X_{P_{3}}=\frac{u_{1}}{u_{2}}+\frac{1}{u_{2} u_{3}}+\frac{1}{u_{3}}=\frac{1+u_{2}+u_{1} u_{3}}{u_{2} u_{3}}, \\
& X_{P_{1}}=\frac{u_{3}}{u_{2}}+\frac{1}{u_{2} u_{1}}+\frac{1}{u_{1}}=\frac{1+u_{2}+u_{1} u_{3}}{u_{2} u_{1}},
\end{aligned}
$$

The submodules of $I_{2}$ are $0, P_{1}, P_{3}, S_{2}$ and $I_{2}$.

$$
X_{I_{2}}=\frac{1}{u_{2}}+\frac{1}{u_{1} u_{3}}+\frac{1}{u_{1} u_{3}}+\frac{1}{u_{1} u_{2} u_{3}}+\frac{u_{2}}{u_{1} u_{3}}=\frac{1+2 u_{2}+u_{2}^{2}+u_{1} u_{3}}{u_{1} u_{2} u_{3}},
$$

The submodules of $S_{1}$ are 0 and $S_{1}$; the submodules of $S_{3}$ are 0 and $S_{3}$.

$$
X_{S_{1}}=\frac{1}{u_{1}}+\frac{u_{2}}{u_{1}}=\frac{1+u_{2}}{u_{1}}, \quad X_{S_{3}}=\frac{1}{u_{3}}+\frac{u_{2}}{u_{3}}=\frac{1+u_{2}}{u_{3}} .
$$

Example 3.2. If $Q$ is a quiver of type $\mathrm{A}_{n}$, and if $M$ is an indecomposable module of $\bmod _{k}(Q)$, then $\chi\left(\operatorname{Gr}_{e}(M)\right)=0$ or 1 . More precisely, the indecomposable $k Q$-modules correspond to connected full subquivers of $Q$. Let $Q_{M}$ be the quiver corresponding to $M$ and let $V_{M}$ be the set of its vertices. Then, the submodules $N$ of $M$ correspond to subsets $V_{N}$ of $V_{M}$ such that the following property holds: $i \in V_{N}$ and $i \rightarrow j \Rightarrow j \in V_{N}$.

Now, if $N$ is a submodule of $M$ with dimension vector $\underline{n}$, then $\operatorname{Gr}_{\underline{n}}(M)$ has only one point and $\chi\left(\mathrm{Gr}_{\underline{n}}(M)\right)=1$.

In the particular case where $Q$ is the equioriented quiver of type $\mathrm{A}_{n}$, the property above implies that, for each indecomposable module $M$, the number of terms in the decomposition of $X_{M}$ is $\operatorname{dim} M+1$.

Example 3.3. We consider the following quiver $Q$ of type $\mathrm{D}_{4}$ :

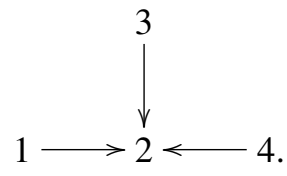

Let $M$ be the indecomposable module with maximal dimension, i.e. $[M]=\left[S_{1}\right]+$ $\left[S_{3}\right]+\left[S_{4}\right]+2\left[S_{2}\right]$. Then, we have $\mathrm{Gr}_{\alpha_{2}} M=\mathbb{P}^{1}$ and so $\chi\left(\mathrm{Gr}_{\alpha_{2}} M\right)=2$. 
The module $M$ has 13 submodules: $0, S_{2}, 2 S_{2}, P_{1}, P_{3}, P_{4}, P_{1}+S_{2}, P_{3}+S_{2}$, $P_{4}+S_{2}, P_{1}+P_{3}, P_{1}+S_{2}, P_{1}+P_{4}, P_{3}+P_{4}, M$. But, $S_{2}$ has "multiplicity" 2 . That gives

$$
X_{M}=\frac{\left(1+u_{2}\right)^{3}+2 u_{1} u_{3} u_{4}+3 u_{1} u_{2} u_{3} u_{4}+u_{1}^{2} u_{3}^{2} u_{4}^{2}}{u_{1} u_{2}^{2} u_{3} u_{4}} .
$$

Let $E_{Q}$ be the $\mathbb{Q}\left[u_{i}, 1 \leq i \leq n\right]$-submodule of $\mathcal{F}$ generated by $X_{M}, M \in$ $\overline{\bmod }_{k}(Q)$, then:

Theorem 3.4. For each quiver $Q$ of type $A-D-E, E_{Q}$ is a subalgebra of $\mathcal{F}$. It identifies with the subalgebra $\mathcal{A}\left(B_{Q}\right)=\mathcal{A}(\Delta)$ of $\mathcal{F}$. Up to this identification, the set of cluster variables of $\mathcal{A}\left(B_{Q}\right)$ is given by $\left\{u_{i}, 1 \leq i \leq n\right\} \cup\left\{X_{M}, M \in \operatorname{Ind}_{k}(Q)\right\}$.

Note that in particular, up to isomorphism, the algebra $E_{Q}$ does not depend on $Q$ but only on $\Delta$.

The subsections below are devoted to the proof of this theorem.

3.2. In order to calculate the Euler-Poincaré characteristic of Grassmannians, we will use the following classical lemma, see [Rei], which is an application of Grothendieck-Lefschetz's fixed point formula for the Frobenius in étale cohomology.

Lemma 3.5. Let $X$ be a variety defined over some ring of algebraic integers. We denote by $X_{\mathbb{C}}$ (resp. $X_{\mathbb{F}_{q}}$ ) the set of $\mathbb{C}$-points (resp. $\mathbb{F}_{q}$-points) of $X$. Suppose that there exists a polynomial $P$ with integral coefficients such that $\left|X_{\mathbb{F}_{q}}\right|=P(q)$ for infinitely many prime powers $q$. Then, the Euler-Poincaré characteristic (with compact support) of $X_{\mathbb{C}}$ is given by $\chi\left(X_{\mathbb{C}}\right)=P(1)$.

Note first that the Grassmannians discussed above are defined over $\mathbb{Z}$. Indeed, Grassmannians of $k$-subspaces are defined by base change from a $\mathbb{Z}$-scheme, [DG80, 3.13]. Moreover, as the $k Q$-modules are defined on $\mathbb{Z}$, it is easily seen that a subspace is a submodule if and only if it verifies $\mathbb{Z}$-linear conditions on the Plücker coordinates of the subspace. Note also that the cardinality of Grassmannians of submodules is given by sums of Hall polynomials. Indeed, for a fixed module $M$ and a fixed dimension vector of a submodule $N$, there is only a finite number of possibilities for the isomorphism classes of $N$ and of the quotient $M / N$, as their dimension vectors are fixed and the quiver is of finite type. Hence the cardinality of a Grassmannian of submodules of fixed dimension vector is a finite sum of the cardinalities of sets of triples $(N, M, M / N)$ where the isomorphism classes are fixed. These cardinalities are known to be polynomials in $q$, called Hall polynomials, see [Rin90].

3.3. We prove here that $E_{Q}$ is a subalgebra of $\mathcal{F}$. Actually, we will prove the following result. 
Proposition 3.6. Fix g in $Q$. For all $M, N$ in $\overline{\bmod }_{k}(Q)$, we have

$$
\chi\left(\operatorname{Gr}_{\underline{g}}(M \oplus N)\right)=\sum_{\underline{e}+\underline{f}=\underline{g}} \chi\left(\operatorname{Gr}_{\underline{e}}(M)\right) \chi\left(\operatorname{Gr}_{\underline{f}}(N)\right) .
$$

By the bilinearity of the Euler form, this proposition implies

Corollary 3.7. For all $M, N$ in $\overline{\bmod }_{k}(Q)$, we have $X_{M} X_{N}=X_{M \oplus N}$. Hence, $E_{Q}$ is a subalgebra of $\mathcal{F}$. It is the $\mathbb{Q}$-subalgebra generated by $\left\{u_{i}, 1 \leq i \leq n\right\} \cup\left\{X_{M}\right.$, $\left.M \in \operatorname{Ind}_{k}(Q)\right\}$.

By Lemma 3.5, Proposition 3.6 can be obtained by counting points on $\mathbb{F}_{q}$ varieties. Set $k=\mathbb{F}_{q}$. Fix two $k Q$-modules $M$ and $N$. Let $\pi: M \oplus N \rightarrow N$ be the projection on the second factor.

Fix a submodule $A$ of $M$ and a submodule $B$ of $N$. Let us introduce

$$
\operatorname{Gr}_{A, B}(M \oplus N):=\{L \in \operatorname{Gr}(M \oplus N), L \cap M=A \text { and } \pi(L)=B\} .
$$

Lemma 3.8. Fix a submodule $A$ of $M$ and a submodule $B$ of $N$. Let $\pi_{A}: M \rightarrow A$ be the canonical projection. There exists a bijection

$$
\operatorname{Hom}_{k}(B, M / A) \rightarrow \mathrm{Gr}_{A, B}(M \oplus N),
$$

which maps the morphism fto $L_{f}=\left\{m+b, m \in M, b \in B, \pi_{A}(m)=f(b)\right\}$.

Proof. The space $L_{f}$ is a submodule of $M \oplus N$ and $\pi\left(L_{f}\right)=B$ by construction. Moreover, $L_{f} \cap M=\operatorname{Ker}\left(\pi_{A}\right)=A$, hence, $L_{f} \in \operatorname{Gr}_{A, B}(M \oplus N)$ and the map is well defined.

To show that the correspondence $f \mapsto L_{f}$ is bijective, define the opposite direction map as follows: let $L \in \mathrm{Gr}_{A, B}(M \oplus N)$. For $b$ in $B$, define $f_{L}(b)=\pi_{A}(m)$ for any $m \in M$ such that $m+b \in L$. Since $L \cap M=A$, this map is well defined. Clearly, both left and right compositions with $f \mapsto L_{f}$ are identity maps, as needed.

Proof of Proposition 3.6. For $g$ in $\mathcal{Q}$, consider the map

$$
\zeta_{\underline{g}}: \operatorname{Gr}_{\underline{g}}(M \oplus N) \rightarrow \coprod_{\underline{e}+\underline{f}=\underline{g}} \operatorname{Gr}_{\underline{e}}(M) \times \operatorname{Gr}_{\underline{f}}(N), L \mapsto(L \cap M, \pi(L)) .
$$

This map is clearly surjective: $\zeta_{g}(A \oplus B)=(A, B)$. Moreover, the lemma above proves that $\zeta_{g}^{-1}(A, B)$ has $q^{[B, M / A]}$ elements. Now, the proposition is a direct consequence of Lemma 3.5. 
3.4. Now, we need to understand the natural set of generators of the algebra $E_{Q}$, $\left\{u_{i}, 1 \leq i \leq n\right\} \cup\left\{X_{M}, M \in \operatorname{Ind}_{k}(Q)\right\}$ by Corollary 3.7. We want to prove that it is precisely the set of cluster variables of $\mathcal{A}\left(B_{Q}\right)$. By construction the variables $u_{i}$, $1 \leq i \leq n$, are cluster variables. Now, for each indecomposable module $M$ in $\bmod _{k}(Q)$, let $\nu(M)$ be the smallest integer such that $\tau^{\nu(M)} M=0$ in $\bmod _{k}(Q)$. We want to prove by induction on $v(M)$ that $X_{M}$ is a cluster variable of $\mathcal{A}\left(B_{Q}\right)$. The case $v(M)=1$ corresponds to the projective case. By Section 2.5 (Proposition 2.6), in this case, we have to prove:

Lemma 3.9. For all $i, 1 \leq i \leq n$, we have

$$
u_{i} X_{P_{i}}=\prod_{i \rightarrow j} X_{P_{j}} \prod_{k \rightarrow i} u_{k}+1 .
$$

Proof. Set $\underline{d}_{i}:=\underline{\operatorname{dim}}\left(P_{i}\right)$. It is known that the radical $\operatorname{Rad} P_{i}$ verifies the following:

(i) $P_{i} / \operatorname{Rad} P_{i}=S_{i}$,

(ii) $M \subset P_{i} \Leftrightarrow M \subset \operatorname{Rad} P_{i}$ or $M=P_{i}$,

(iii) $\operatorname{Rad} P_{i}=\oplus_{i \rightarrow j} P_{j}$.

By (i), we have

$$
X_{\operatorname{Rad} P_{i}}=\sum_{\underline{e}} \chi\left(\mathrm{Gr}_{\underline{e}} \operatorname{Rad} P_{i}\right) \prod_{l} u_{l}^{-\left\langle\underline{e}, \alpha_{l}\right\rangle-\left\langle\alpha_{l}, \underline{d}_{i}-\alpha_{i}-\underline{e}\right\rangle} .
$$

Using the fact that the Euler form satisfies

$$
\left\langle\alpha_{k}, \alpha_{i}\right\rangle= \begin{cases}1 & \text { if } k=i \\ -1 & \text { if } k \rightarrow i \\ 0 & \text { else }\end{cases}
$$

one gets

$$
X_{\operatorname{Rad} P_{i}}=\sum_{\underline{e}} \chi\left(\operatorname{Gr}_{\underline{e}} \operatorname{Rad} P_{i}\right)\left(\prod_{l} u_{l}^{-\left\langle\underline{e}, \alpha_{l}\right\rangle-\left\langle\alpha_{l}, \underline{d}_{i}-\underline{e}\right\rangle}\right)\left(\prod_{k \rightarrow i} u_{k}^{-1}\right) u_{i} .
$$

By (ii), we have

$$
X_{P_{i}}=\sum_{\underline{e}} \chi\left(\mathrm{Gr}_{\underline{e}} \operatorname{Rad} P_{i}\right) \prod_{l} u_{l}^{-\left\langle\underline{e}, \alpha_{l}\right\rangle-\left\langle\alpha_{l}, \underline{d}_{i}-\underline{e}\right\rangle}+u_{i}^{-1} .
$$

Comparing with (30) gives

$$
X_{P_{i}}=X_{\operatorname{Rad} P_{i}}\left(\prod_{k \rightarrow i} u_{k}\right) u_{i}^{-1}+u_{i}^{-1} .
$$

The lemma is now a consequence of (iii) and Corollary 3.7. 
3.5. We prove here the induction discussed in Section 3.4. What we need to prove is that for every non projective indecomposable $k Q$-module $N$, if $X_{\tau N}$ is a cluster variable of $\mathcal{A}(\Delta)$, then $X_{N}$ is also a cluster variable. By Section 2.5 (Proposition 2.6), what we have to prove is this result.

Proposition 3.10. Suppose that $M, N$ are indecomposable modules and

$$
0 \longrightarrow M \stackrel{\iota}{\longrightarrow} B \stackrel{\pi}{\longrightarrow} N \longrightarrow 0
$$

is an almost split exact sequence, then $X_{M \oplus N}=X_{M} X_{N}=X_{B}+1$.

Remark that $M \oplus N$ and $B$ are the middle terms $Y$ of respectively a split sequence and an almost split sequence $0 \rightarrow M \rightarrow Y \rightarrow N \rightarrow 0$. The reader may view the formula above as a "difference" between split and almost split. The proof of the proposition is an adaptation of the proof in Section 3.3 in the almost split case.

Proof. Set $\underline{m}=\underline{\operatorname{dim}} M, \underline{n}=\underline{\operatorname{dim}} N$. Recall that $M=\tau N$. We have

$$
X_{M \oplus N}=\sum_{\underline{e}} \chi\left(\operatorname{Gr}_{\underline{e}}(M \oplus N)\right) \prod_{i} u_{i}^{-\left\langle\underline{e}, \alpha_{i}\right\rangle-\left\langle\alpha_{i}, \underline{m}+\underline{n}-\underline{e}\right\rangle} .
$$

By Lemma 3.5, $X_{M \oplus N}$ can be seen as a polynomial of $\mathbb{Z}\left[u_{i}^{ \pm 1}\right][q]$ evaluated at $q=1$ :

$$
X_{M \oplus N}=\left.\left(\sum_{L} \prod_{i} u_{i}^{-\left\langle\underline{\operatorname{dim}} L, \alpha_{i}\right\rangle-\left\langle\alpha_{i}, \underline{m}+\underline{n}-\underline{\operatorname{dim}} L\right\rangle}\right)\right|_{q=1},
$$

where $L$ runs over the set of submodules of $M \oplus N$ and $k=\mathbb{F}_{q}$. In this formula the term corresponding to the submodule $L=0 \oplus N$ in $M \oplus N$ is

$$
\prod_{i} u_{i}^{-\left\langle\underline{n}, \alpha_{i}\right\rangle-\left\langle\alpha_{i}, \underline{m}\right\rangle}=1
$$

by the Serre duality formula.

As in the proof of Proposition 3.6, our proposition follows from lemma below.

Lemma 3.11. Consider the map

$$
\zeta_{\underline{g}}: \operatorname{Gr}_{\underline{g}}(B) \rightarrow \coprod_{\underline{e}+\underline{f}=\underline{g} \underline{\underline{g}}} \operatorname{Gr}_{\underline{e}}(M) \times \operatorname{Gr}_{\underline{f}}(N), L \mapsto\left(\iota^{-1}(L), \pi(L)\right),
$$

where $\iota$ and $\pi$ are the morphisms in (33). The fiber of a point $(A, C)$ is empty if $(A, C)=(0, N)$, and is an affine space of dimension $[C, M / A]$ if not. 
Proof. Let us prove the case $(A, C)=(0, N)$. Suppose that $L \subset B, \pi(L)=N$ and $\iota^{-1}(L)=0$. Then, $\pi$ provides an isomorphism $L \simeq N$. This implies that the map $\pi$ splits but this is impossible since $\pi$ is the surjection of an almost split sequence.

Suppose now $(A, C) \neq(0, N)$. If $C$ is not equal to $N$, then, the "almost split" property implies that $\pi$ has a section $C \rightarrow B$. This case is analog to the split case which was considered in Section 3.3 and the proof is similar.

It remains to prove the case where $A \neq 0$ and $C=N$. The Auslander-Reiten theorem yields a duality

$$
\Theta: \operatorname{Hom}(A, M) \otimes \operatorname{Ext}^{1}(N, A) \rightarrow \operatorname{Ext}^{1}(N, M) \simeq k .
$$

Note that $\Theta$ is just composition of morphisms in the derived category.

Let $i_{A} \in \operatorname{Hom}(A, M)$ be the canonical embedding. By the duality, there exists $\eta$ in $\operatorname{Ext}^{1}(N, A)$ such that $\Theta\left(i_{A} \otimes \eta\right) \in \operatorname{Ext}^{1}(N, M)$ does not vanish. By a classical result, this implies that there exist $E, \varphi$ and a commutative diagram

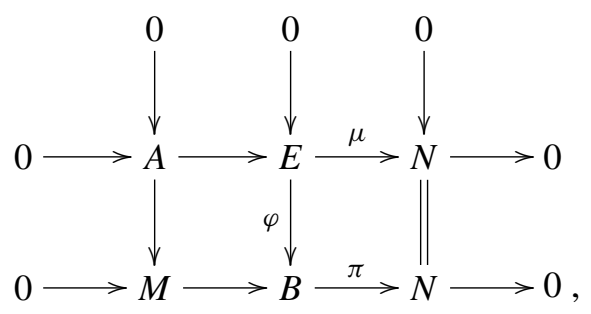

where the top exact sequence corresponds to $\eta$. The morphism $\varphi$ is mono by an easy diagram chasing.

Hence, $E \in \zeta_{g}^{-1}(A, N)$ and the fiber is non empty. In this case, it is a well-known fact that the fiber is an affine space with a simple transitive action of $\operatorname{Hom}(N, M / A)$. We give the proof for completion.

Let $\mu: E \rightarrow N$ as in the diagram above and $\pi_{A}: M \rightarrow M / A$ the canonical surjection. We consider the map $\operatorname{Hom}(N, M / A) \rightarrow \zeta_{g}^{-1}(A, N)$, defined by $f \mapsto$ $E_{f}:=\left\{\iota(m)+\varphi(e), m \in M, e \in E, \pi_{A}(m)=\bar{f} \mu(e)\right\}$. The space $E_{f}$ is a submodule of $B$. We start with the following remark. Suppose that

$$
\iota\left(m^{\prime}\right)+\varphi\left(e^{\prime}\right)=\iota(m)+\varphi(e), \quad m, m^{\prime} \in M, e, e^{\prime} \in E .
$$

Applying $\pi$ gives $e^{\prime}-e \in \iota(A)$ and then $\pi_{A}(m)=\pi_{A}\left(m^{\prime}\right)$. In particular, this easily implies that $E_{f}$ is in the fiber $\zeta_{g}^{-1}(A, N)$.

In order to prove that the correspondence $f \mapsto E_{f}$ is bijective, define the opposite direction map as follows: let $D \in \zeta_{\underline{g}}^{-1}(A, N)$, for $n$ in $N$, set $f_{D}(n)=\pi_{A}(m)$, where $\iota(m)+\varphi(e) \in D$ and $\mu(e)=n$. By the remark above, $f_{D}$ is a well-defined element of $\operatorname{Hom}(N, M / A)$. 
We have $E_{f_{D}}=D$. Indeed, it is enough to prove the inclusion, as both modules have same dimension vector $g$. Let $x$ in $E_{f_{D}}$, hence we have a decomposition $x=$ $\iota(m)+\varphi(e)$, with $\pi_{A}(m)=\bar{f}_{D}(\mu(e))$. By construction of $f_{D}$, we have $\pi_{A}(m)=$ $f_{D} \mu e=\pi_{A}\left(m^{\prime}\right)$, with $\iota\left(m^{\prime}\right)+\varphi(e) \in D$. Hence, $x=\iota(m)+\varphi(e)=\iota\left(m^{\prime}\right)+\varphi(e) \in$ $D$, as desired.

We have $f_{E_{f}}=f$. Indeed, $f_{E_{f}}(n)=\pi_{A}(m)$, where $\iota(m)+\varphi(e) \in E_{f}$ and $\mu(e)=n$. By definition of $E_{f}$ we have: $\iota(m)+\varphi(e)=\iota\left(m^{\prime}\right)+\varphi\left(e^{\prime}\right)$, and $\pi_{A}\left(m^{\prime}\right)=$ $f \mu\left(e^{\prime}\right)$. So, by the remark above,

$$
f_{E_{f}}(n)=\pi_{A}(m)=\pi_{A}\left(m^{\prime}\right)=f \mu\left(e^{\prime}\right)=f \mu(e)=f(n) .
$$

This ends the proof.

\section{A conjecture in the multiplicity-free case}

4.1. Let $Q$ be a Dynkin quiver. In particular the category of modules over $Q$ is hereditary. Let $M$ be an indecomposable object in the category of modules over the quiver $Q$. Assume that $M$ is multiplicity-free, that is $\operatorname{dim}\left(M_{i}\right) \leq 1$ for all $i$. This implies that a submodule $N$ of $M$ is determined by its dimension vector. In these cases, the Grassmannian is either empty or a point.

Then Formula (14) for the cluster corresponding to $Q$ and the cluster variable corresponding to $M$ can be restated as follows.

Proposition 4.1. One has

$$
X_{M}=\frac{1}{\prod_{i \in M} u_{i}} \sum_{N \subset M} \prod_{i \in N}\left(\prod_{i \rightarrow j} u_{j}\right) \prod_{i \in M / N}\left(\prod_{j \rightarrow i} u_{j}\right),
$$

where the sum runs over submodules $N$ of $M$ and the index $j$ in the inner products runs over the set of vertices of $Q$.

Proof. Indeed, Formula (14) can be reformulated, using injective and projective resolutions, the definition of the Euler form and the known Euler-Poincaré characteristic of Grassmannians, as the following expression:

$$
X_{M}=\sum_{N \subset M} \frac{\left[P_{1}^{N}\right]}{\left[P_{0}^{N}\right]} \frac{\left[I_{1}^{M / N}\right]}{\left[I_{0}^{M / N}\right]},
$$

where

$$
0 \longrightarrow P_{1}^{N} \longrightarrow P_{0}^{N} \longrightarrow N \longrightarrow 0
$$


and

$$
0 \longrightarrow M / N \longrightarrow I_{0}^{M / N} \longrightarrow I_{1}^{M / N} \longrightarrow 0
$$

are projective and injective resolutions and the brackets mean replacing the direct sum of projective modules $P_{i}$ or injective modules $I_{i}$ by the corresponding product of variables $u_{i}$.

Let us fix a submodule $N$ of $M$ and denote by $\mathrm{S}(N)$ the set of sources of the quiver underlying $N$.

Using the hypotheses that the module $N$ is multiplicity-free and that the quiver $Q$ is Dynkin of finite type, hence a tree, one can describe completely the minimal projective resolution of $N$. The first step $P_{0}^{N}$ is the direct sum of all projective modules $P_{j}$ for $j \in \mathrm{S}(N)$. By the hereditary property, the dimension vector of the second step $P_{1}^{N}$ is known. Its support is made of some isolated elements inside $N$ and some branches starting just outside $N$. Each relative sink $j$ in $N \backslash \mathrm{S}(N)$ contributes to $P_{1}^{N}$ by the direct sum of $\mathrm{N}(j)-1$ copies of $P_{j}$ where $\mathrm{N}(j)$ is the number of arrows inside $N$ with target $j$. Each of the branches corresponds to a projective $P_{j}$ for some $j$ outside $N$ with an arrow $i \rightarrow j$ for some $i \in N$.

From this description of the projective resolution of $N$, one gets that

$$
\frac{\left[P_{1}^{N}\right]}{\left[P_{0}^{N}\right]}=\frac{\prod_{i \in N}\left(\prod_{j \notin N, i \rightarrow j} u_{j}\right) \prod_{j \in N \backslash \mathrm{S}(N)} u_{j}^{\mathrm{N}(j)-1}}{\prod_{j \in \mathrm{S}(N)} u_{j}} .
$$

This becomes

$$
\prod_{i \in N}\left(\prod_{j \notin N, i \rightarrow j} u_{j}\right) \prod_{j \in N} u_{j}^{\mathrm{N}(j)-1} .
$$

Then it follows that

$$
\frac{\left[P_{1}^{N}\right]}{\left[P_{0}^{N}\right]}=\frac{\prod_{i \in N}\left(\prod_{i \rightarrow j} u_{j}\right)}{\prod_{j \in N} u_{j}},
$$

where the index $j$ in the numerator product runs over the set of vertices of $Q$. A similar argument for the injective resolution of $M / N$ completes the proof.

4.2. Let us now consider a quiver $Q$ of finite cluster type, as introduced in [FZ03a] and studied in [CCS04]. This can be one of the quivers of Dynkin type considered before, but many other quivers arise in the mutation process starting from a Dynkin quiver. These quivers can be defined from the matrix $B$ of a seed $(\underline{x}, B)$ in a simplylaced finite reduced cluster algebra by the rule that there is an arrow from $i$ to $j$ if and only if one has $b_{i j}=1$. This rule was already used for the Dynkin quivers in Section 2.4.

Then it is expected in general and known in type A [CCS04] that there is a correspondence between cluster variables (other than the initial ones) for the seed 
associated to $Q$ and indecomposables of the category of modules over $Q$ with some relations. In this bijection, the denominators of the cluster variables should be described by the dimension vectors of the indecomposables.

Although the precise relations are not known outside of type A, a conjecture for them has been made in [CCS04] and it is usually a simple task to check in any particular case that the proposed relations have the expected properties.

Assuming now that the proposed relations are correct or that the correct relations are known, let us propose a formula for the cluster variables associated to multiplicityfree indecomposables. Let $M$ be a multiplicity-free indecomposable object in the category of modules over the quiver $Q$ with relations. Abusing notation, we will denote a submodule $N$ of $M$ and its support by the same letter.

Let $\mathrm{E}_{M}$ be the set of arrows of $Q$ between vertices of $M$ such that the associated morphism in $M$ is zero. For $e$ in $\mathrm{E}_{M}$, let $s(e)$ and $t(e)$ be the source and target of $e$. We will display later an example of cluster quiver and a module over it in type $\mathrm{D}_{4}$ where $\mathrm{E}_{M}$ is not empty.

Conjecture 4.2. The cluster variable $X_{M}$ has the following expression:

$$
\frac{1}{\prod_{i \in M} u_{i}} \sum_{N \subset M} \frac{\prod_{i \in N}\left(\prod_{i \rightarrow j} u_{j}\right) \prod_{i \in M / N}\left(\prod_{j \rightarrow i} u_{j}\right)}{\prod_{i \notin M, i \rightarrow M, M \rightarrow i} u_{i} \prod_{e \in \mathrm{E}_{M}} u_{s(e)} u_{t(e)}}
$$

where the sum runs over submodules $N$ of $M$. Here $i \rightarrow M$ means that there exists $k$ in $M$ and an arrow $i \rightarrow k$. The meaning of $M \rightarrow i$ is similar. The index $j$ in the inner products runs over the set of vertices of $Q$.

One can recognize in the left factor of this formula the expression for what should be the denominator of the cluster variable. Therefore the remaining part should be a formula for the numerator.

In the case of a Dynkin quiver $Q$, as $M$ is assumed indecomposable, there can not be any vanishing edge in the support of $M$, hence $\mathrm{E}_{M}$ is empty. As $Q$ is a tree, there is no vertex outside $M$ with arrows in $Q$ to $M$ and from $M$. Hence Formula (40) is a special case of Conjecture 4.2 and this conjecture holds in the case of Dynkin quivers.

4.3. Let us give two simple examples of Formula (47) for non-Dynkin cluster quivers. Let us consider first the following cluster quiver $Q$ of type $\mathrm{A}_{3}$ :

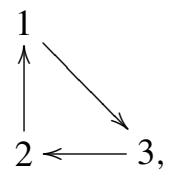


with the relations $f_{1,2} f_{2,3}=f_{2,3} f_{3,1}=f_{3,1} f_{1,2}=0$ according to [CCS04]. Let $M$ be the indecomposable multiplicity-free module with support $\{1,2\}$. It has 3 submodules: 0, $M$ and a submodule with support $\{1\}$. Then Formula (47) gives

$$
X_{M}=\left(\frac{1}{u_{1} u_{2}}\right) \frac{(1)\left(u_{3} u_{1}\right)+\left(u_{3}\right)\left(u_{3}\right)+\left(u_{3} u_{2}\right)(1)}{u_{3}}=\frac{u_{1}+u_{2}+u_{3}}{u_{1} u_{2}},
$$

which is the correct expression.

Let us consider now the following cluster quiver $Q$ of type $\mathrm{D}_{4}$ :



with relations $f_{1,2} f_{2,4}=f_{1,3} f_{3,4}$ and $f_{2,4} f_{4,1}=f_{4,1} f_{1,2}=f_{4,1} f_{1,3}=f_{1,3} f_{3,4}=0$, as conjectured in [CCS04]. One can easily compute the indecomposable objects and the Auslander-Reiten quiver for the category of modules on this quiver with these relations. One can then check that the dimension vectors of the indecomposable modules correspond to the denominators of the cluster variables when expressed in this cluster. This proves in this particular case some conjectures made in [CCS04]. Let $M$ be the following indecomposable module:

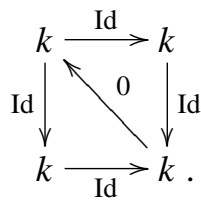

Note that the set $\mathrm{E}_{M}$ contains the diagonal arrow.

The module $M$ has 6 submodules: $0, M$ and submodules with support $\{4\},\{2,4\}$, $\{3,4\}$ and $\{2,3,4\}$. Then using Formula (47), one gets that $X_{M}$ is equal to

$$
\frac{(1)\left(u_{1}^{2} u_{2} u_{3} u_{4}\right)+\left(u_{1}\right)\left(u_{1}^{2} u_{4}\right)+(1+1)\left(u_{1} u_{4}\right)\left(u_{1} u_{4}\right)+\left(u_{1} u_{4}^{2}\right)\left(u_{4}\right)+\left(u_{1} u_{2} u_{3} u_{4}^{2}\right)(1)}{\left(u_{1} u_{2} u_{3} u_{4}\right)\left(u_{1} u_{4}\right)},
$$

which simplifies to the correct expression:

$$
X_{M}=\frac{\left(u_{1}+u_{4}\right)^{2}+u_{2} u_{3}\left(u_{1}+u_{4}\right)}{u_{1} u_{2} u_{3} u_{4}} .
$$

\section{Coxeter-Conway friezes}

We give here an interpretation of Coxeter-Conway friezes [CC73], which follows directly from the main theorem. 
5.1. Following Conway and Coxeter, we construct a frieze from a triangulation of the $(n+3)$-gon. The construction is the following.

We consider a triangulation $T$ of the $(n+3)$-gon, i.e. a maximal set of non crossing diagonals of the polygon. Note that each maximal set has exactly $n$ diagonals. To each vertex $k, k \in \mathbb{Z} /(n+3) \mathbb{Z}$, of the polygon, let $d_{k}$ be the number of diagonals of $T$ containing the vertex $k$. Set $m_{k}=d_{k}+1$. We construct a frieze filled with numbers in the following way.

We place a bottom row $\mathrm{R}_{0}$ filled with 1 . Then, we place above it (in a shifted way, see Example 5.1 below) a row filled with $m_{k}, k \in \mathbb{Z} /(n+3) \mathbb{Z}$. Then, we fill further shifted rows above such that each diamond

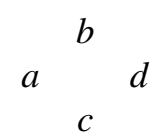

verifies $a d=1+b c$, until we reach again a row filled with 1 only.

Example 5.1. Consider the triangulation $T$ of Figure 1. Then, the corresponding frieze is

$\begin{array}{llllllllllllll}1 & & 1 & & 1 & & 1 & & 1 & & 1 & & 1 & \\ & 1 & & 3 & & 2 & & 1 & & 3 & & 2 & & 1 \\ 1 & & 2 & & 5 & & 1 & & 2 & & 5 & & 1 & \\ & 1 & & 3 & & 2 & & 1 & & 3 & & 2 & & 1 \\ 1 & & 1 & & 1 & & 1 & & 1 & & 1 & & 1 . & \end{array}$

Now, we make a connection with another construction. In [CCS04], the authors define for each triangulation $T$ of the $(n+3)$-gon a quiver $Q_{T}$ in the following way.

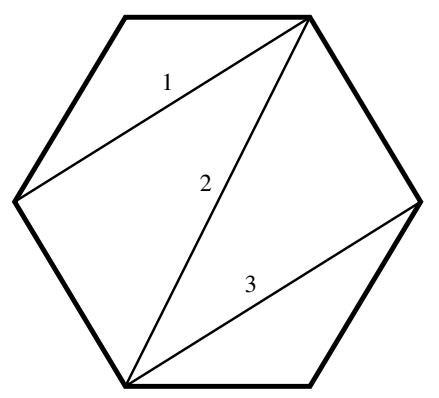

Figure 1

Let $D_{i}, 1 \leq i \leq n$, be the diagonals of $T$. The set of vertices of the quiver $Q_{T}$ is $\{1, \ldots, n\}$, and $i \rightarrow j$ if and only if $D_{i}$ and $D_{j}$ are edges of a triangle in $T$ and if the 
angle from $D_{i}$ to $D_{j}$ is counterclockwise. We can choose the triangulation such that $Q_{T}$ is any orientation of the Dynkin diagram of type $\mathrm{A}_{n}$. In this case, we can define the categories $\bmod _{k}(Q)$ and $\mathcal{C}$ as before.

For any indecomposable object $M$ of $\mathcal{C}$, let $\mathrm{x}_{M}$ be the following number

$$
\mathrm{x}_{M}=\left.X_{M}\right|_{u_{1}=\cdots=u_{n}=1} .
$$

We have the proposition:

Proposition 5.2. Let $T$ be a triangulation of the $(n+3)$-gon such that the associated quiver $Q_{T}$ is an orientation of the Dynkin diagram of type $A_{n}$. Let $\gamma_{T}$ be obtained from the AR-quiver of $\Gamma_{Q_{T}}$ by replacing each $M$ by the number $\mathrm{x}_{M}$. Then, $\gamma_{T}$ is the Coxeter-Conway frieze associated to $T$.

Remark 5.3. The reader can check the proposition on an example by comparing Example 3.1 and Example 5.1.

Proof. We sketch the proof of the proposition. In [CCS04, par. 5], the authors define a bijection from the set of diagonals of the $(n+3)$-gon to the set of indecomposable objects of $\mathcal{C}$. And moreover, the objects of the first row of the AR-quiver $\Gamma_{\mathcal{C}}$ of $\mathcal{C}$ correspond to diagonals of type $[k-1, k+1], k \in \mathbb{Z} /(n+3) \mathbb{Z}$. Let $M_{k}$ be the object corresponding to $[k-1, k+1]$. As $T$ is a triangulation, two cases can occur. Either, $M_{k}$ is a diagonal of $T$, or $M_{k}$ intersect $T$ non trivially. By [CCS04, par. 5] $M_{k}$ is an object of $\bmod _{k}(Q)$ if and only if we are in the second case. Moreover, in this case the quiver associated to $M_{k}$ as in Example 3.2 is equioriented. Indeed, the diagonals of $T$ cutting $[k-1, k+1]$ can be totally ordered in a counterclockwise way, as shown in Figure 2. So, by 3.2, $M_{k}$ has exactly $\operatorname{dim} M_{k}+1=\mathrm{x}_{M_{k}}$ submodules. Moreover, the dimension of $M_{k}$ is $\left[\oplus_{i} P_{i}, M_{k}\right]=\sum_{i}\left[M_{k}, S P_{i}\right]^{1}$ which, by [CCS04], is exactly the

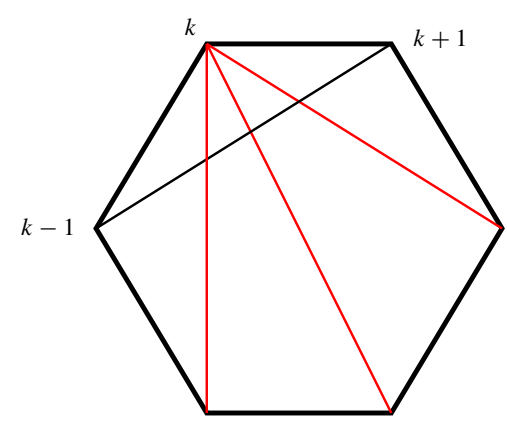

Figure 2 
number of diagonals of $T$ intersected by the corresponding diagonal $[k-1, k+1]$. So, $\mathbf{x}_{M_{k}}=m_{k}$. Now, the diamond relations correspond to exchange relations as in Proposition 3.10. We obtain the result by induction on the rows.

\section{References}

[ARS95] M. Auslander, I. Reiten, and S. O. Smalø, Representation theory of Artin algebras. Cambridge Stud. Adv. Math. 36, Cambridge University Press, Cambridge 1995. Zbl 0834.16001 MR 1314422

[BFZ05] A. Berenstein, S. Fomin, and A. Zelevinsky, Cluster algebras. III. Upper bounds and double Bruhat cells. Duke Math. J. 126 (2005), 1-52. Zbl 02147024 MR 2110627

$\left[\mathrm{BMR}^{+}\right]$A. Buan, R. J. Marsh, M. Reineke, I. Reiten, and G. Todorov, Tilting theory and cluster combinatorics. arXiv:math.RT/0402054, 2004.

[CC73] J. H. Conway and H. S. M. Coxeter, Triangulated polygons and frieze patterns. Math. Gaz. 57 (1973), 87-94. Zbl 0285.05028 MR 0461269

[CCS04] P. Caldero, F. Chapoton, and R. Schiffler, Quivers with relations arising from clusters ( $A_{n}$ case). Trans. Amer. Math. Soc. 358 (2006), 1347-1364. Zbl 02237892

[CK] P. Caldero and B. Keller, From triangulated categories to cluster algebras. Preprint, math.RT/0506018, 2005.

[DG80] M. Demazure and P. Gabriel, Introduction to algebraic geometry and algebraic groups. Translated from the French by J. Bell, North-Holland Math. Stud. 39, NorthHolland Publishing Co., Amsterdam 1980. Zbl 0431.14015 MR 0563524

[FG03] V. V. Fock and A. B. Goncharov, Moduli spaces of local systems and higher Teichmüller theory. Preprint, math.AG/0311149, 2003.

[FZ02] S. Fomin and A. Zelevinsky, Cluster algebras. I. Foundations. J. Amer. Math. Soc. 15 (2002), 497-529 (electronic). Zbl 1021.16017 MR 1887642

[FZ03a] S. Fomin and A. Zelevinsky, Cluster algebras. II. Finite type classification. Invent. Math. 154 (2003), 63-121. Zbl 1054.17024 MR 2004457

[FZ03b] S. Fomin and A. Zelevinsky, $Y$-systems and generalized associahedra. Ann. of Math. (2) 158 (2003), 977-1018. Zbl 1057.52003 MR 2031858

[Gab80] P. Gabriel, Auslander-Reiten sequences and representation-finite algebras. In Representation theory, I (Proc. Workshop, Carleton Univ., Ottawa, Ont., 1979), Lecture Notes in Math. 831, Springer-Verlag, Berlin 1980, 1-71. Zbl 0445.16023 MR 0607140

[Kel] B. Keller, On triangulated orbit categories. Doc. Math., J. DMV 10 (2005), 551-581. Zbl 02238184

[MRZ03] R. Marsh, M. Reineke, and A. Zelevinsky, Generalized associahedra via quiver representations. Trans. Amer. Math. Soc. 355 (2003), 4171-4186. Zbl 1042.52007 MR 1990581

[Rei] M. Reineke, Counting rational points of quiver moduli. Preprint, math.AG/0505389, 2005. 
[Rin90] C. M. Ringel, Hall algebras and quantum groups. Invent. Math. 101 (1990), 583-591. Zbl 0735.16009 MR 1062796

[Sco06] J. S. Scott, Grassmannians and Cluster Algebras. Proc. London Math. Soc. 92 (2006), 345-380. Zbl 05014382 MR 2205721

Received November 2, 2004; revised April 28, 2005

Philippe Caldero, Institut Camille Jordan, Université Claude Bernard Lyon I, 69622 Villeurbanne Cedex, France

E-mail: caldero@math.univ-lyon1.fr

Frédéric Chapoton, Institut Camille Jordan, Université Claude Bernard Lyon I, 69622 Villeurbanne Cedex, France

E-mail: chapoton@math.univ-lyon1.fr 